\title{
Infertility Counseling
}

\author{
Beth N Laisure
}

\begin{abstract}
Infertility, whether reasons known or unexplained, has been shown to cause a high amount of stress for people who receive this diagnosis. Increased emotional stress can lead to more of a decreased chance of conception, along with other medical problems. Around the world infertility counseling is becoming a new focus for medical professionals to work combined with fertility treatments and help couples conceive. Couples using medicines to help with conception, undergoing intrauterine insemination, and in vitro fertilization who have added counseling to treatment have shown an increase in conception rates.
\end{abstract}

Keywords: Counseling infertility, Stress and infertility, Webbased counseling.

How to cite this article: Laisure BN. Infertility Counseling. Int J Infertil Fetal Med 2017;8(1):32-35.

Source of support: Nil

Conflict of interest: None

Date of received: $25-8-2016$

Date of acceptance: 30-9-2016

Date of publication: May 2017

\section{INFERTILITY COUNSELING}

In today's society, the age that couples are starting to try to conceive has increased. The majority of couples are waiting until a later age to marry and therefore, start a family. When problems with fertility arise at a later stage of couples' lives, the stress related to the increased urgency to conceive is seen by medical professionals. Couples age advances when beginning trying to conceive, the chances of conception decrease. This leads to couples turning to assisted reproductive technology (ART) sooner and with much more urgency than seen in the past. This author became interested in this subject of infertility stress after being diagnosed with unexplained infertility and wondering what research shows as the best way to reduce infertility-related stress.

Smith et al ${ }^{1}$ suggest that chronic stress, depression, and anxiety which can develop in couples who have

\footnotetext{
Registered Nurse

Department of Berkeley Medical Center, West Virginia University Healthcare in Martinsburg, West Virginia, USA

Corresponding Author: Beth N Laisure, Department of Berkeley Medical Center, West Virginia University Healthcare in Martinsburg, West Virginia, USA, Phone: +15403270928, e-mail: bn10220@gmail.com
}

trouble conceiving can lead to more problems with conception. Similarly, Abedinia et $\mathrm{al}^{2}$ report in estimations of anxiety, $86.8 \%$ of women reported a high level of anxiety with trouble conceiving. Multiple fertility clinics and reproductive endocrinologists have now begun to suggest counseling of some form when couples are beginning ART. Marcus et $\mathrm{al}^{3}$ report certain countries have mandated that couples going through ART be offered counseling sessions as part of their infertility regimen. These findings have led this author to question: In couples undergoing infertility treatments does attending standard counseling sessions decrease the stress and anxiety derived from ART, and therefore, increase the success rate of conception compared to those without counseling sessions over a period of a year? This review of literature will also try to determine the best methods for reducing stress/anxiety / depression for couples undergoing ART treatments.

\section{RETRIEVAL OF LITERATURE}

An internet search was conducted through EBSCO host database for randomized control trials related to infertility and counseling. Inclusion criteria for articles set at publication dates of 2000 to 2015 and full text articles only. Keywords used in the search included stress and infertility, counseling for infertility, and web-based infertility counseling. These articles were chosen for inclusion in this review because of the research topics and being randomized control trials. Articles had to include those with variables of decreasing stress during infertility treatments.

\section{REVIEW OF LITERATURE}

In the United Kingdom, fertility clinics are mandated to offer counseling to couples undergoing ART. Marcus et $\mathrm{al}^{3}$ sought to identify the number of patients who received counseling, how helpful the counseling was, and the reasons behind some couples choosing not to receive counseling. This article's aim was to understand the views of patients attending different fertility centers on fertility counseling. The authors noted that most trials concerning fertility are biased in that they are only testing one fertility clinics results. The authors sent out patient self-reported questionnaires to all registered users of a fertility website. The questionnaire was returned by 244 users all going through a different array of ARTs, such as in vitro fertilization (IVF) or intracytoplasmic sperm injection (ICSI) using own or donor egg/sperm. 
The results of Marcus et $\mathrm{al}^{3}$ survey concluded that out of the patients who received counseling during ART, over $50 \%$ viewed the counseling as helpful. The majority of the people in this study, $70 \%$, did not receive counseling. The reasons behind not receiving counseling sessions were feeling as if they could cope on their own, counseling was not offered, or did not feel it would be beneficial. This study concluded that a very low number, $30 \%$, actually took counseling that was offered, but the author states that this is congruent with other similar studies. Other studies have found that most people do not want counseling from a stranger but would rather rely on close family and friends for stress support.

Marcus et $\mathrm{al}^{3}$ report the limitations of their study as only targeting those infertile couples who have internet access and this may not be representative of all infertile couples due to others having lack of internet access. The authors propose that there is not a significant difference in couples without internet access. The authors also state that $21 \%$ of the people sampled did not receive counseling because it was not offered; they voice the problems with this, since in the United Kingdom counseling is mandated to at least be offered in fertility clinics. Although this article found that $50 \%$ of couples reported the importance of infertility counseling, it does not test the outcomes of the counseling, such as conception/birth rates in these couples. This article is valuable in establishing the importance of counseling to over half of the subjects tested and so can conclude on the helpfulness of infertility counseling.

The second article in this review is an article by Mori. ${ }^{4}$ This is a randomized control trial of supportive stress management in women undergoing the early stages of fertility treatment. Participants in this study were less than or equal to 35 years of age and undergoing general fertility treatments for less than 2 years. The study was conducted at seven different institutions with experiment and control groups being randomly assigned. Recruitment was obtained through people working at the institutions who had no direct involvement in the study. Participants in the study were divided into two groups, programs A and B. All participants in the study were given a booklet called, "A guidebook for women facing the challenge of infertility: You are not alone - self-management against stress." Those participants in program A were additionally given notebooks for a stress diary, a relaxation diary, a social support network, and a stress calendar. Participants in program $\mathrm{B}$ received no further materials. The investigator sent questionnaires to all individuals once per month to rate their self-management of stress at that time. They were also contacted once per month by telephone or email to answer any questions.
A total of 140 women met the inclusion criteria for this study, with a total of 103 women completing the study at the 3-month end. The author developed three hypotheses for testing with the study: The incidence of depression and anxiety using the Hospital Anxiety and Depression Scale was predicted to be lower in the experimental group vs the control group, the health status score using Medical Outcomes Study Short Form 36-item Health Survey (SF36) was predicted to be higher for experimental group, and the satisfaction score using Visual Analogue Scale was predicted to be higher in experimental group. Out of the three hypotheses tested with this study, only the health status score with SF-36 showed a significant difference between experimental and control groups. The experimental group which received the homework notebook reported that this tool helped the participants recognize and take action against one's stress level. The participants through follow-up phone calls and interactive communication learned new coping strategies to stress. The other two hypotheses were not supported by the study.

The author reports that the limitations of this study are many. The amount of women who withdrew during the 3-month trial in the experimental group was 20.8\% and almost four times more than the control group. The author believes the drop-out rate was from the homework assignments being time-consuming for the participants. Other limitations of this study included only including women who have had fertility complications for 2 years or less, most other studies show significant levels of anxiety or depression with longer time of infertility. The author concluded that although not all hypotheses were proven true in this study, the effects of infertility on depression, overall health status, and satisfaction is something which needs more research. Two of the subscales of the SF-36 did show positive effects of a support program for women who were struggling with fertility. Although this small number does not seem significant, the author does state that future research is warranted.

The next article in this review is by Sexton et al. ${ }^{5}$ The authors of this study sought to find if a web-based intervention would decrease the stress related to infertility treatments. The authors randomly assigned women recruited from fertility clinics to intervention or waitlist control groups. Those in the intervention group were given passwords to an internet website and asked to participate in web-directed activities. The activities on the website were based on coping with infertility (CWI), which is an evidence-based intervention to decrease stress, anxiety, depression, grief, and relationship stress. The authors developed a web-based coping with infertility (WCWI) using only the depression and stress/anxiety modules from CWI. The authors chose to only have the intervention period of 2 weeks to quickly evaluate the results and 
provide feedback of necessity of bibliotherapy interventions for women undergoing infertility treatments.

Results of this study suggest that the WCWI-based treatments significantly lowered the depression/stress the group faced with infertility. The limitations to this study were that only women were tested with the trial, the women within the study were all of high economic class, and the investigators remain uncertain of the webbased treatments actually decreasing the stress/anxiety these women felt because of the nature of the study. The authors conclude that although the limitations are many, the preliminary results warrant further research on the subject and believe that for women undergoing fertility treatments, a web-based self-help intervention could be a way to decrease anxiety/stress related to diagnosis.

The fourth article in this review by Haemmerli et al ${ }^{6}$ used a randomized control trial to study the effects of internet-based support for infertile patients. This study provided an 8-week intervention using the Child Wish Online Coaching program to improve mental health in patients undergoing fertility treatments. The program consisted of self-help guide, text-based contact with a therapist, and forums for conversations with other members. The participants (those in the intervention group) were assigned 13 different sessions each with different focuses to be completed online. The participants could move through the sessions at their own pace. The intervention lasted 8 weeks, pre- and posttesting was used to identify usefulness of the intervention, and participants were also given a 5-month follow-up evaluation.

Although the authors of this article did not find statistical correlation to those participants in the intervention group sustaining higher pregnancy rates, it did, however, find that the program did affect the depression level on those who completed the intervention. Over $80 \%$ of the participants viewed the intervention as positive or very positive. This data led the authors to conclude that webbased interventions for those experiencing infertility has potential to decrease the stress related to ART. The authors state the limitations of this study were that bias could be seen in the fact that the participants of this study expressed interest in the internet-based treatment, fertility treatment types (IVF, intrauterine insemination, etc.) were not differentiated, and participants reported shorter duration of infertility than most other studies aim to study.

The last article in this review by Abedinia et $\mathrm{al}^{2}$ sought to find the effects of a psychological intervention on infertile couples' quality of life. The authors treated participants in the intervention group with six to eight sessions of psychotherapy and medication treatment of fluoxetin (20-60 mg doses) before infertility treatment began. The authors began the study by performing the Beck Depression Inventory (BDI) on 638 couples at an infertility clinic. The couples scoring high on the BDI were then invited to participate in the study. In the study, 140 couples were chosen to participate and were divided into groups using randomization. The groups consisted of preinfertility treatment intervention and during fertility treatment intervention.

The results of this study were that those participants who received the treatments before going through infertility treatments had a pregnancy rate of 47.1 vs $7.1 \%$ in the control group. The authors concluded that psychological interventions can increase pregnancy rate and therefore, improve mental health among infertile patients. The limitations of this study were that only one fertility clinic was used which could lead to bias and the participants had to rate on the depression scale as being depressed before being included in the study. Although some infertile couples could exhibit stress / anxiety with infertility diagnosis which could affect treatment, they may not rank high enough on the BDI for depression to be included in the study.

\section{SYNTHESIS OF FINDINGS}

This review was conducted to answer the question whether fertility counseling is able to increase pregnancy rates and decrease psychological distress in infertile couples. All of the studies in this review concluded that decreasing depression/stress in infertile couples is beneficial and stressed the need of counseling when patients are undergoing fertility treatments. Only one study ${ }^{2}$ in this review noted pregnancy rate change in intervention vs control groups. Other studies ${ }^{5,6}$ were able to show significance in treating stress/anxiety or depression in infertile couples with new technologies, such as internet-based counseling. Although the study ${ }^{6}$ showed that no statistical correlation was found between counseling and pregnancy rates, the participants in the study rated the web-based interventions positively in increasing the support they felt with treatment. The studies ${ }^{3,4}$ that used in-person counseling sessions to decrease stress related to infertility concluded that the participants found the counseling to be beneficial in decreasing stress/depression. Tools, such as the BDI have shown to yield positive effects in assessment of stress as related to infertility and ART. ${ }^{2,3}$

\section{GAPS AND FUTURE DIRECTIONS}

Cultural and societal values in today's societies have led to an increase in stress related to infertility treatments. ${ }^{2-5}$ The stress/anxiety/depression that couples' feel during infertility treatments needs to be treated alongside medical treatments for infertility. The articles in this review used different techniques with the common goal of decreasing the amount of stress/depression on those 
who are diagnosed with infertility during treatment. The gaps in the available knowledge of these articles included that no article found a significant correlation between reducing stress and increasing pregnancy rates, except one. Although most research warrants the need for decreasing stress/depression in this population of people,$^{2-6}$ the commonality of an infertile diagnosis has not led to an evidence-based approach to solving the problem of infertility stress. The need, therefore, becomes a way to diagnose stress/anxiety in infertility couples and evidence to support best practices in treating infertile couples. All the articles voiced the need to decrease stress in this population of people, none were successful in reporting statistically significant way to achieve that goal. ${ }^{2-6}$ Most of the participants throughout each study found the stress reduction techniques involved as helpful or useful to them. Gaps also include different stages of treatments in which patients are in; Are couples who are just starting to see a specialist regarding infertility at the same stress/depression level as couples who are in the third round of IVF?

The future of research regarding decreasing stress / depression related to infertility should be in delineating a common strategy for stress reduction and clinical trials to show evidence of the positive effects of infertility counseling. Evidence-based practice for stress reduction in this population of patients needs to be the top priority of clinicians treating these patients. Congruent stress management care whether with self-help techniques to reduce stress through web-learning or in-center sessions needs that it start on the first day of a patient seeking ART. ${ }^{7}$ If stress management through internet-based counseling, support groups, counseling sessions occurred at the onset of infertility diagnosis, the stress/anxiety that increases with advancing treatments (IVF or ICSI) could potentially be thwarted in early stages, preparing those who suffer with infertility to manage their stress. ${ }^{7}$ This review has concluded the known importance of identifying strategies by clinicians to reduce stress / anxiety associated with infertility treatments. Furthermore, continual research to identify best practices for managing stress in infertile couples seeking treatment is a high priority.

\section{REFERENCES}

1. Smith CA, Ussher JM, Perz J, Carmady B, de Lacey S. The effect of acupuncture on psychosocial outcomes for women experiencing infertility: a pilot randomized control trial. J Altern Complement Med 2011 Oct;17(10):923-930.

2. Abedinia N, Ramezanzadeh F, Noorbala AA. Effects of a psychological intervention on quality of life in infertile couples. J Fam Reprod Health 2009 Oct;3(3):87-93.

3. Marcus D, Marcus H, Marcus N, Appleton T, Marcus S. Infertility counseling - an internet-based survey. Hum Fertil 2007 Jun;10(2):111-116.

4. Mori A. Supporting stress management for women undergoing the early stages of fertility treatment: a cluster-randomized controlled trial. Jpn J Nurs Sci 2009 Jun;6(1):37-49.

5. Sexton M, Byrd M, O'Donohue W, Jacobs N. Web-based treatment for infertility-related psychological distress. Arch Womens Ment Health 2010 Aug;13(4):347-358.

6. Haemmerli K, Znoj H, Berger T. Internet-based support for infertile patients: a randomized controlled study. J Behav Med 2010 Apr;33(2):135-146.

7. Katz S. MINDFUL CARE: an integrative tool to guide holistic treatment in enhancing fertility. Perspect Psychiatr Care 2010;44(3):207-210. 